\title{
On cross-intersecting uniform sub-families of hereditary families
}

\author{
Peter Borg \\ Department of Mathematics \\ University of Malta \\ Msida MSD 2080, Malta \\ p.borg.02@cantab.net
}

Submitted: Sep 15, 2009; Accepted: Apr 5, 2010; Published: Apr 19, 2010

Mathematics Subject Classification: 05 D05

\begin{abstract}
A family $\mathcal{H}$ of sets is hereditary if any subset of any set in $\mathcal{H}$ is in $\mathcal{H}$. If two families $\mathcal{A}$ and $\mathcal{B}$ are such that any set in $\mathcal{A}$ intersects any set in $\mathcal{B}$, then we say that $(\mathcal{A}, \mathcal{B})$ is a cross-intersection pair $($ cip $)$. We say that a cip $(\mathcal{A}, \mathcal{B})$ is simple if at least one of $\mathcal{A}$ and $\mathcal{B}$ contains only one set. For a family $\mathcal{F}$, let $\mu(\mathcal{F})$ denote the size of a smallest set in $\mathcal{F}$ that is not a subset of any other set in $\mathcal{F}$. For any positive integer $r$, let $[r]:=\{1,2, \ldots, r\}, 2^{[r]}:=\{A: A \subseteq[r]\}, \mathcal{F}^{(r)}:=\{F \in \mathcal{F}:|F|=r\}$.

We show that if a hereditary family $\mathcal{H} \subseteq 2^{[n]}$ is compressed, $\mu(\mathcal{H}) \geqslant r+s$ with $r \leqslant s$, and $(\mathcal{A}, \mathcal{B})$ is a cip with $\emptyset \neq \mathcal{A} \subset \mathcal{H}^{(r)}$ and $\emptyset \neq \mathcal{B} \subset \mathcal{H}^{(s)}$, then $|\mathcal{A}|+|\mathcal{B}|$ is a maximum if $(\mathcal{A}, \mathcal{B})$ is the simple $\operatorname{cip}\left(\{[r]\},\left\{B \in \mathcal{H}^{(s)}: B \cap[r] \neq \emptyset\right\}\right)$; Frankl and Tokushige proved this for $\mathcal{H}=2^{[n]}$. We also show that for any $2 \leqslant r \leqslant s$ and $m \geqslant r+s$ there exist (non-compressed) hereditary families $\mathcal{H}$ with $\mu(\mathcal{H})=m$ such that no $\operatorname{cip}(\mathcal{A}, \mathcal{B})$ with a maximum value of $|\mathcal{A}|+|\mathcal{B}|$ under the condition that $\emptyset \neq \mathcal{A} \subset \mathcal{H}^{(r)}$ and $\emptyset \neq \mathcal{B} \subset \mathcal{H}^{(s)}$ is simple (we prove that this is not the case for $r=1$ ), and we suggest two conjectures about the extremal structures in general.
\end{abstract}

\section{Introduction}

We shall use small letters such as $x$ to denote elements of a set or positive integers, capital letters such as $X$ to denote sets, and calligraphic letters such as $\mathcal{F}$ to denote families (i.e. sets whose members are sets themselves). Unless otherwise stated, it is to be assumed that sets and families are finite.

$\mathbb{N}$ is the set $\{1,2, \ldots\}$ of positive integers. For $m, n \in \mathbb{N}$ with $m \leqslant n$, the set $\{i \in$ $\mathbb{N}: m \leqslant i \leqslant n\}$ is denoted by $[m, n]$, and if $m=1$ then we also write $[n]$. The power set $\{A: A \subseteq X\}$ of a set $X$ is denoted by $2^{X}$, and $\{A \subseteq X:|A|=r\}$ is denoted by $\left(\begin{array}{c}X \\ r\end{array}\right)$. 
We next develop some notation for certain sets and families defined on a family $\mathcal{F} \subseteq$ $2^{X}$. Let $U(\mathcal{F})$ denote the union of all sets in $\mathcal{F}$. Let $\mathcal{F}^{(r)}:=\{F \in \mathcal{F}:|F|=r\}$. For a set $Y$, let $\mathcal{F}(Y):=\{F \in \mathcal{F}: F \cap Y \neq \emptyset\}$ and $\mathcal{F}[Y]:=\{F \in \mathcal{F}: Y \subseteq F\}$. For a single-element set $\{y\}$, we may abbreviate the notation $\mathcal{F}(\{y\})$ to $\mathcal{F}(y)$, and we set $\mathcal{F}\langle y\rangle:=\{F \backslash\{y\}: F \in \mathcal{F}(y)\}$.

For $i, j \in[n]$, let $\Delta_{i, j}: 2^{2^{[n]}} \rightarrow 2^{2^{[n]}}$ be the compression operation (see [4]) defined by

$$
\Delta_{i, j}(\mathcal{F}):=\left\{\delta_{i, j}(F): F \in \mathcal{F}, \delta_{i, j}(F) \notin \mathcal{F}\right\} \cup\left\{F \in \mathcal{F}: \delta_{i, j}(F) \in \mathcal{F}\right\},
$$

where $\delta_{i, j}: 2^{[n]} \rightarrow 2^{[n]}$ is defined by

$$
\delta_{i, j}(F):= \begin{cases}(F \backslash\{j\}) \cup\{i\} & \text { if } i \notin F \text { and } j \in F \\ F & \text { otherwise. }\end{cases}
$$

A family $\mathcal{F}$ is said to be

- a hereditary family (or an ideal or a downset) if all subsets of any set in $\mathcal{F}$ are in $\mathcal{F}$;

- uniform if the sets in $\mathcal{F}$ have the same size;

- intersecting if any set in $\mathcal{F}$ intersects any other set in $\mathcal{F}$;

- centred if the sets in $\mathcal{F}$ contain a common element;

- compressed if $\mathcal{F} \subseteq 2^{[n]}$ and $\Delta_{i, j}(\mathcal{F})=\mathcal{F}$ for any $i, j \in[n]$ with $i<j$;

- compressed with respect to $x \in U(\mathcal{F})$ if $\Delta_{x, y}(\mathcal{F})=\mathcal{F}$ for any $y \in U(\mathcal{F})$.

Two families $\mathcal{A}$ and $\mathcal{B}$ are said to be cross-intersecting if any set in $\mathcal{A}$ intersects any set in $\mathcal{B}$. We say that $(\mathcal{A}, \mathcal{B})$ is a cross-intersection pair (cip) if $\mathcal{A}$ and $\mathcal{B}$ are cross-intersecting. We say that a $\operatorname{cip}(\mathcal{A}, \mathcal{B})$ is simple if at least one of $\mathcal{A}$ and $\mathcal{B}$ contains only one set.

Hilton and Milner [7] proved that if $r \leqslant n / 2$ and $\mathcal{A}, \mathcal{B}$ are non-empty cross-intersecting sub-families of $\left(\begin{array}{c}{[n]} \\ r\end{array}\right)$, then $|\mathcal{A}|+|\mathcal{B}| \leqslant\left(\begin{array}{c}n \\ r\end{array}\right)-\left(\begin{array}{c}n-r \\ r\end{array}\right)+1=\left|\mathcal{A}_{0}\right|+\left|\mathcal{B}_{0}\right|$, where $\mathcal{A}_{0}$ is $\{[r]\}$ and $\mathcal{B}_{0}$ is $\left\{B \in\left(\begin{array}{c}{[n]} \\ r\end{array}\right): B \cap[r] \neq \emptyset\right\}$. A streamlined proof of this result was later obtained by Simpson [10] by means of the compression (also known as shifting) technique introduced in the seminal paper [4] (see [5] for a good survey on the uses of this technique in extremal set theory). Frankl and Tokushige [6] instead used the Kruskal-Katona Theorem [8, 9] to establish the following extension.

Theorem 1.1 (Frankl and Tokushige [6]) If $r \leqslant s, n \geqslant r+s$, and $(\mathcal{A}, \mathcal{B})$ is a cip with $\emptyset \neq \mathcal{A} \subseteq\left(\begin{array}{c}{[n]} \\ r\end{array}\right)$ and $\emptyset \neq \mathcal{B} \subseteq\left(\begin{array}{c}{[n]} \\ s\end{array}\right)$, then $|\mathcal{A}|+|\mathcal{B}| \leqslant\left(\begin{array}{c}n \\ s\end{array}\right)-\left(\begin{array}{c}n-r \\ s\end{array}\right)+1=\left|\mathcal{A}_{0}\right|+\left|\mathcal{B}_{0}\right|$, where $\left(\mathcal{A}_{0}, \mathcal{B}_{0}\right)$ is the simple cip $\left(\{[r]\},\left\{B \in\left(\begin{array}{c}{[n]} \\ s\end{array}\right): B \cap[r] \neq \emptyset\right\}\right)$.

In this paper we are interested in cip's $(\mathcal{A}, \mathcal{B})$ having a maximum value of $|\mathcal{A}|+|\mathcal{B}|$ under the condition that both $\mathcal{A}$ and $\mathcal{B}$ are non-empty uniform sub-families of a hereditary family $\mathcal{H}$. Note that Theorem 1.1 deals with the special case when $\mathcal{H}$ is the power set $2^{[n]}$, which is the simplest example of a hereditary family. It is easy to see that a family is hereditary if and only if it is a union of power sets. There are many interesting examples of hereditary families, such as the family of independent sets of a graph or matroid.

Before stating our results, we shall introduce a few more definitions. 
We say that a set $M$ is $\mathcal{F}$-maximal if $M$ is not a subset of any set in $\mathcal{F} \backslash\{M\}$. We denote the size of a smallest $\mathcal{F}$-maximal set in $\mathcal{F}$ by $\mu(\mathcal{F})$.

For a family $\mathcal{F}$, we denote the set $\{(\mathcal{A}, \mathcal{B}):(\mathcal{A}, \mathcal{B})$ is a cip with a maximum value of $|\mathcal{A}|+|\mathcal{B}|$ under the condition that $\emptyset \neq \mathcal{A} \subset \mathcal{F}^{(r)}$ and $\left.\emptyset \neq \mathcal{B} \subset \mathcal{F}^{(s)}\right\}$ by $C(\mathcal{F}, r, s)$.

Using the compression technique, we generalise Theorem 1.1 as follows.

Theorem 1.2 If $r \leqslant s, n \geqslant r+s$, and $\mathcal{H}$ is a compressed hereditary sub-family of $2^{[n]}$ with $\mu(\mathcal{H}) \geqslant r+s$, then the simple cip $\left(\{[r]\},\left\{B \in \mathcal{H}^{(s)}: B \cap[r] \neq \emptyset\right\}\right)$ is in $C(\mathcal{H}, r, s)$.

Theorem 1.1 is the case $\mathcal{H}=2^{[n]}$, in which $[n]$ is the only $\mathcal{H}$-maximal set in $\mathcal{H}$ and hence $\mu(\mathcal{H})=n$. Note that we cannot relax the condition that $\mu(\mathcal{H}) \geqslant r+s$. Indeed, if $\mathcal{H}=2^{[n]}$ and $s \leqslant \mu(\mathcal{H})<r+s$, then any set in $\mathcal{H}^{(r)}=\left(\begin{array}{c}{[n]} \\ r\end{array}\right)$ intersects any set in $\mathcal{H}^{(s)}=\left(\begin{array}{c}{[n]} \\ s\end{array}\right)$ (since $n=\mu(\mathcal{H})<r+s$ ), and hence $\left(\mathcal{H}^{(r)}, \mathcal{H}^{(s)}\right)$ is the only cip in $C(\mathcal{H}, r, s)$. Note that if $\mathcal{H}=2^{[n]}$ and $\mu(\mathcal{H})<s$, then $C(\mathcal{H}, r, s)=\emptyset$ (since $n=\mu(\mathcal{H})<s$ and hence $\left.\mathcal{H}^{(s)}=\emptyset\right)$.

Remark 1.3 One of the central problems in extremal set theory is the famous Chvátal Conjecture [2], which claims that at least one of the largest intersecting sub-families of any hereditary family $\mathcal{H}$ is centred. Chvátal [3] proved his conjecture for the case when $\mathcal{H}$ is compressed. Snevily [11] improved Chvátal's result to the case when $\mathcal{H}$ is compressed with respect to an element of $U(\mathcal{H})$. In the next section we show that no similar improvement can be made to Theorem 1.2 for $r \geqslant 2$; more precisely, we show that for any $2 \leqslant r \leqslant s$ and $m \geqslant r+s$ there are hereditary families $\mathcal{H}$ with $\mu(\mathcal{H})=m$ such that $\mathcal{H}$ is compressed with respect to an element of $U(\mathcal{H})$ and no $\operatorname{cip}$ in $C(\mathcal{H}, r, s)$ is simple. We then suggest two conjectures about the structure of at least one of the cip's in $C(\mathcal{H}, r, s)$ for any hereditary family $\mathcal{H}$ with $\mu(\mathcal{H}) \geqslant r+s$.

For $r=1$ we do have the desired general result.

Theorem 1.4 If $\mathcal{H}$ is a hereditary family with $\mu(\mathcal{H}) \geqslant 1+s$, then $C(\mathcal{H}, 1, s)$ has a simple $\operatorname{cip}\left(\mathcal{A}_{0}, \mathcal{B}_{0}\right)$ with $\mathcal{A}_{0}=\{\{x\}\}$ and $\mathcal{B}_{0}=\left\{B \in \mathcal{H}^{(s)}: x \in B\right\}$ for some $x \in U(H)$.

Proof. Let $(\mathcal{A}, \mathcal{B}) \in C(\mathcal{H}, 1, s)$. Suppose $|\mathcal{A}|=1$. Then, since $\mathcal{A} \subset \mathcal{H}^{(1)}, \mathcal{A}=\{\{x\}\}$ for some $x \in U(\mathcal{H})$. Since $\mathcal{B} \subset \mathcal{H}^{(s)}$ and $|\mathcal{A}|+|\mathcal{B}|$ is a maximum (under the cross-intersection condition), $\mathcal{B}$ must consist of all the sets in $\mathcal{H}^{(s)}$ which contain $x$.

Now suppose $|\mathcal{A}|>1$. Let $Z:=\{z \in U(\mathcal{H}):\{z\} \in \mathcal{A}\}$; so $|Z|=|\mathcal{A}|$ and hence $|Z|>1$. Since every set in $\mathcal{B}$ must intersect every (single-element) set in $\mathcal{A}$, we clearly have $\mathcal{B} \subseteq \mathcal{H}^{(s)}[Z]\left(=\left\{H \in \mathcal{H}^{(s)}: Z \subseteq H\right\}\right)$. Let $B \in \mathcal{B}$. Since every (single-element) set in $\mathcal{A}$ must intersect $B$, we have $Z \subseteq B$ and hence $|Z| \leqslant s$. Let $x \in Z$ and let $M$ be an $\mathcal{H}$-maximal set in $\mathcal{H}$ such that $B \subset M$. Then $|M| \geqslant 1+s($ as $|M| \geqslant \mu(\mathcal{H})), Z \subset M$ (as $Z \subseteq B$ ), and $\left(\begin{array}{c}M \\ s\end{array}\right) \subseteq \mathcal{H}^{(s)}$ (as $\mathcal{H}$ is hereditary). Now let $\left(\mathcal{A}_{0}, \mathcal{B}_{0}\right)$ be the simple cip $\left(\{\{x\}\}, \mathcal{H}^{(s)}(x)\right)$. Since $(\mathcal{A}, \mathcal{B}) \in C(\mathcal{H}, 1, s),\left|\mathcal{A}_{0}\right|+\left|\mathcal{B}_{0}\right| \leqslant|\mathcal{A}|+|\mathcal{B}|$. Also, 


$$
\begin{aligned}
\left|\mathcal{A}_{0}\right|+\left|\mathcal{B}_{0}\right| & =1+\left|\mathcal{H}^{(s)}(x)\right| \\
& \geqslant 1+\left|\mathcal{H}^{(s)}[Z]\right|+\left|\left\{A \in\left(\begin{array}{c}
M \\
s
\end{array}\right): x \in A,|A \cap Z|=|Z|-1\right\}\right| \\
& =1+\left|\mathcal{H}^{(s)}[Z]\right|+\left(\begin{array}{c}
|Z|-1 \\
|Z|-2
\end{array}\right)\left(\begin{array}{c}
|M|-|Z| \\
s-(|Z|-1)
\end{array}\right) \\
& \geqslant|Z|+\left|\mathcal{H}^{(s)}[Z]\right|=|\mathcal{A}|+\left|\mathcal{H}^{(s)}[Z]\right| \geqslant|\mathcal{A}|+|\mathcal{B}| .
\end{aligned}
$$

So we actually have $\left|\mathcal{A}_{0}\right|+\left|\mathcal{B}_{0}\right|=|\mathcal{A}|+|\mathcal{B}|$, and hence $\left(\mathcal{A}_{0}, \mathcal{B}_{0}\right) \in C(\mathcal{H}, 1, s)$.

The above result will be used in the proof of Theorem 1.2. It is easy to see from its proof that if $\mu(\mathcal{H})>1+s$, then any $(\mathcal{A}, \mathcal{B})$ in $C(\mathcal{H}, 1, s)$ is a simple cip as in the result.

\section{A construction and two conjectures}

The following is the proof of the claim in Remark 1.3.

Proposition 2.1 Let $2 \leqslant l+1 \leqslant r \leqslant s, m \geqslant r+s$ and $p>\left(\left(\begin{array}{c}m-l \\ s\end{array}\right)-\left(\begin{array}{c}m-r \\ s\end{array}\right)+1\right) /\left(\begin{array}{c}m-l \\ r-l\end{array}\right)$. For each $i \in[p]$, let $M_{i}:=[l] \cup[(i-1)(m-l)+l+1, i(m-l)+l]$. Let $\mathcal{E}=\bigcup_{i=1}^{p} 2^{M_{i}}$. Then $\mathcal{E}$ is hereditary, $\mathcal{E}$ is compressed with respect to $1, \mu(\mathcal{E})=m$, and no $\operatorname{cip}$ in $C(\mathcal{E}, r, s)$ is simple.

Proof. It is straightforward that $\mathcal{E}$ is hereditary, $\mathcal{E}$ is compressed with respect to 1 , and $\mu(\mathcal{E})=\left|M_{1}\right|=\ldots=\left|M_{p}\right|=m$. Let $(\mathcal{A}, \mathcal{B})$ be a simple cip with $\emptyset \neq \mathcal{A} \subseteq \mathcal{E}^{(r)}$ and $\emptyset \neq \mathcal{B} \subseteq \mathcal{E}^{(s)}$. Let $L:=[l], \mathcal{A}_{1}:=\left\{L \cup C: C \in\left(\begin{array}{c}M_{i} \backslash L \\ r-l\end{array}\right)\right.$ for some $\left.i \in[p]\right\}, \mathcal{B}_{1}=\mathcal{E}^{(s)}(L)$ $\left(=\left\{E \in \mathcal{E}^{(s)}: E \cap L \neq \emptyset\right\}\right)$. Since $\left(\mathcal{A}_{1}, \mathcal{B}_{1}\right)$ is a non-simple cip with $\emptyset \neq \mathcal{A}_{1} \subseteq \mathcal{E}^{(r)}$ and $\emptyset \neq \mathcal{B}_{1} \subseteq \mathcal{E}^{(s)}$, the result follows if we show that $|\mathcal{A}|+|\mathcal{B}|<\left|\mathcal{A}_{1}\right|+\left|\mathcal{B}_{1}\right|$.

Let $R:=[r], \mathcal{A}_{0}:=\{R\}, \mathcal{B}_{0}:=\mathcal{E}^{(s)}(R)$. We will show that

$$
|\mathcal{A}|+|\mathcal{B}| \leqslant\left|\mathcal{A}_{0}\right|+\left|\mathcal{B}_{0}\right|
$$

Let us first assume this. Note that $\mathcal{B}_{0}$ is the disjoint union of $\mathcal{B}_{1}$ and the family $\mathcal{R}$ of sets in $\mathcal{E}^{(s)}$ that intersect $R$ but not $L$. Since $R$ is a subset of $M_{1}$ but not a subset of any other set $M_{i}$, we clearly have $\mathcal{R}=\left\{A \in\left(\begin{array}{c}M_{1} \backslash L \\ s\end{array}\right): A \cap(R \backslash L) \neq \emptyset\right\}$. We have

$$
\begin{aligned}
\left(\left|\mathcal{A}_{1}\right|+\left|\mathcal{B}_{1}\right|\right)-(|\mathcal{A}|+|\mathcal{B}|) & \geqslant\left(\left|\mathcal{A}_{1}\right|+\left|\mathcal{B}_{1}\right|\right)-\left(\left|\mathcal{A}_{0}\right|+\left|\mathcal{B}_{0}\right|\right) \quad(\text { by }(1)) \\
& =\left(\left|\mathcal{A}_{1}\right|+\left|\mathcal{B}_{1}\right|\right)-\left(\left|\mathcal{A}_{0}\right|+\left|\mathcal{B}_{1}\right|+|\mathcal{R}|\right)=\left|\mathcal{A}_{1}\right|-\left|\mathcal{A}_{0}\right|-|\mathcal{R}| \\
& =p\left(\begin{array}{c}
m-l \\
r-l
\end{array}\right)-\left(\begin{array}{c}
m-l \\
s
\end{array}\right)+\left(\begin{array}{c}
m-r \\
s
\end{array}\right)-1 \\
& >0 \quad \text { (by choice of } p)
\end{aligned}
$$

and hence $|\mathcal{A}|+|\mathcal{B}|<\left|\mathcal{A}_{1}\right|+\left|\mathcal{B}_{1}\right|$ as required. 
We now prove (1). Suppose $\mathcal{A}$ contains only one set $A$. Then $\mathcal{B} \subseteq \mathcal{E}^{(s)}(A)$. Since $l<r$ and $M_{i} \cap M_{j}=L$ for any distinct $i$ and $j$ in $[p]$, there is a unique $k$ in $[p]$ such that $A \subset M_{k}$, and it is therefore easy to see that $\left|\mathcal{E}^{(s)}(A)\right| \leqslant\left|\mathcal{B}_{0}\right|$; so (1) holds in this case. Now suppose $|\mathcal{A}|>1$. Then, since $(\mathcal{A}, \mathcal{B})$ is a simple cip, $\mathcal{B}$ contains only one set $B$ and $\mathcal{A} \subseteq \mathcal{E}^{(r)}(B)$. Let $S:=[s], \mathcal{C}_{0}:=\mathcal{E}^{(r)}(S), \mathcal{D}_{0}:=\{S\}$. Similarly to the above, it is easy to see that $\left|\mathcal{E}^{(r)}(B)\right| \leqslant\left|\mathcal{C}_{0}\right|$; so $|\mathcal{A}|+|\mathcal{B}| \leqslant\left|\mathcal{C}_{0}\right|+\left|\mathcal{D}_{0}\right|$. If $r=s$ then $\left|\mathcal{C}_{0}\right|+\left|\mathcal{D}_{0}\right|=\left|\mathcal{A}_{0}\right|+\left|\mathcal{B}_{0}\right|$ and hence (1) holds again. Suppose $r<s$. For each $i \in[p]$, let $\mathcal{F}_{i}:=\left(\begin{array}{c}M_{i} \\ s\end{array}\right)$ and $\mathcal{G}_{i}:=\left(\begin{array}{c}M_{i} \\ r\end{array}\right)$. Since $R \subset M_{1}$ and $R \cap M_{i}=S \cap M_{i}=L$ for each $i \in[2, p]$, we clearly have $\left|\mathcal{B}_{0}\right|=\left|\mathcal{F}_{1}(R)\right|+\sum_{i=2}^{p}\left|\mathcal{F}_{i}(L)\right|$ and $\left|\mathcal{C}_{0}\right|=\left|\mathcal{G}_{1}(S)\right|+\sum_{i=2}^{p}\left|\mathcal{G}_{i}(L)\right|$. We have $\left|\mathcal{G}_{1}(S)\right|<\left|\mathcal{F}_{1}(R)\right|$ since

$$
\begin{aligned}
& \left|\mathcal{F}_{1}(R)\right|-\left|\mathcal{G}_{1}(S)\right|=\left(\left(\begin{array}{c}
m \\
s
\end{array}\right)-\left(\begin{array}{c}
m-r \\
s
\end{array}\right)\right)-\left(\left(\begin{array}{c}
m \\
r
\end{array}\right)-\left(\begin{array}{c}
m-s \\
r
\end{array}\right)\right) \\
& =\left(\left(\begin{array}{c}
m \\
s
\end{array}\right)-\left(\begin{array}{c}
m \\
r
\end{array}\right)\right)-\left(\left(\begin{array}{c}
m-r \\
s
\end{array}\right)-\left(\begin{array}{c}
m-s \\
r
\end{array}\right)\right) \\
& =\left(\begin{array}{c}
m \\
r
\end{array}\right)\left(\frac{r !(m-r) \ldots(m-s+1)}{s !}-1\right)-\left(\begin{array}{c}
m-s \\
r
\end{array}\right)\left(\frac{r !(m-r) \ldots(m-s+1)}{s !}-1\right)>0 .
\end{aligned}
$$

By a similar calculation, we obtain that $\left|\mathcal{G}_{i}(L)\right|<\left|\mathcal{F}_{i}(L)\right|$ for each $i \in[2, p]$. So we have

$$
\left|\mathcal{C}_{0}\right|+\left|\mathcal{D}_{0}\right|=\left|\mathcal{G}_{1}(S)\right|+\sum_{i=2}^{p}\left|\mathcal{G}_{i}(L)\right|+1<\left|\mathcal{F}_{1}(R)\right|+\sum_{i=2}^{p}\left|\mathcal{F}_{i}(L)\right|+1=\left|\mathcal{A}_{0}\right|+\left|\mathcal{B}_{0}\right|
$$

and hence, since $|\mathcal{A}|+|\mathcal{B}| \leqslant\left|\mathcal{C}_{0}\right|+\left|\mathcal{D}_{0}\right|$, (1) holds again.

Something common to the $\operatorname{cip}\left(\mathcal{A}_{1}, \mathcal{B}_{1}\right)$ in the above proof and the extremal structures determined in Theorems 1.2 and 1.4 is that the first family in the pair is centred. We conjecture that there always exist cip's $(\mathcal{A}, \mathcal{B})$ with $\mathcal{A}$ centred that are extremal under the conditions we have been considering, where by extremal we mean that $|\mathcal{A}|+|\mathcal{B}|$ is a maximum.

Conjecture 2.2 (Weak Form) If $r \leqslant s$ and $\mathcal{H}$ is a hereditary family with $\mu(\mathcal{H}) \geqslant r+s$, then for some $\left(\mathcal{A}_{0}, \mathcal{B}_{0}\right) \in C(\mathcal{H}, r, s), \mathcal{A}_{0}$ is centred.

Conjecture 2.3 (Strong Form) If $r \leqslant s$ and $\mathcal{H}$ is a hereditary family with $\mu(\mathcal{H}) \geqslant r+$ $s$, then there exists a set $H$ in $\mathcal{H}$ with $1 \leqslant|H| \leqslant r$ such that for some $\left(\mathcal{A}_{0}, \mathcal{B}_{0}\right) \in C(\mathcal{H}, r, s)$, $\mathcal{A}_{0}=\left\{A \in \mathcal{H}^{(r)}: H \subseteq A\right\}$ and $\mathcal{B}_{0}=\left\{B \in \mathcal{H}^{(s)}: B \cap H \neq \emptyset\right\}$.

Note that the families $\mathcal{A}_{1}$ and $\mathcal{B}_{1}$ in the proof of Proposition 2.1 have the structure of $\mathcal{A}_{0}$ and $\mathcal{B}_{0}$ in the above conjecture.

\section{Some tools}

This section provides the main tools we need for the proof of Theorem 1.2. We start with a crucial lemma concerning the levels of a hereditary family (see [1, Corollary 3.2]). 
Lemma 3.1 (Borg [1]) If $\mathcal{H}$ is a hereditary family and $r<s \leqslant \mu(\mathcal{H})-r$, then

$$
\left|\mathcal{H}^{(r)}\right| \leqslant \frac{\left(\begin{array}{c}
s \\
s-r
\end{array}\right)}{\left(\begin{array}{c}
\mu(\mathcal{H})-r \\
s-r
\end{array}\right)}\left|\mathcal{H}^{(s)}\right| .
$$

The following is our second important lemma, which purely concerns the parameter $\mu(\mathcal{F})$ of a family $\mathcal{F}$.

Lemma 3.2 Let $\emptyset \neq \mathcal{F} \subseteq 2^{[n]}$ and $a \in[n]$. Let $\mathcal{D}:=\mathcal{F} \backslash \mathcal{F}(a)$ and $\mathcal{E}:=\mathcal{F} \backslash \mathcal{F}(n)$.

(i) If $\mathcal{F}(a) \neq \emptyset$, then $\mu(\mathcal{F}\langle a\rangle) \geqslant \mu(\mathcal{F})-1$.

(ii) If $\mathcal{F}$ is hereditary, then $\mu(\mathcal{D}) \geqslant \mu(\mathcal{F})-1$.

(iii) If $\mathcal{F}$ is compressed and $[n] \notin \mathcal{F}$, then $\mu(\mathcal{E}) \geqslant \mu(\mathcal{F})$.

Proof. Suppose $\mathcal{F}(a) \neq \emptyset$. Let $M$ be an $\mathcal{F}\langle a\rangle$-maximal set in $\mathcal{F}\langle a\rangle$. Then $M^{\prime}:=M \cup\{a\}$ is an $\mathcal{F}$-maximal set in $\mathcal{F}$. So $|M|=\left|M^{\prime}\right|-1 \geqslant \mu(\mathcal{F})-1$. Hence (i).

Suppose $\mathcal{F}$ is hereditary. Then, since $\mathcal{F} \neq \emptyset, \emptyset \in \mathcal{F}$. So $\mathcal{D} \neq \emptyset$. Let $M$ be a $\mathcal{D}$ maximal set in $\mathcal{D}$. Suppose also that $|M|<\mu(\mathcal{F})$. So $M$ is not $\mathcal{F}$-maximal, and hence there exists a set $M^{\prime} \in \mathcal{F}(a)$ such that $M \subset M^{\prime}$ and $M^{\prime}$ is $\mathcal{F}$-maximal. Since $\mathcal{F}$ is hereditary, $M^{\prime \prime}:=M^{\prime} \backslash\{a\} \in \mathcal{F}$. Since $M$ is $\mathcal{D}$-maximal and $M \subseteq M^{\prime \prime} \in \mathcal{D}, M=M^{\prime \prime}$. So $M^{\prime}=M \cup\{a\}$. Therefore $|M|=\left|M^{\prime}\right|-1 \geqslant \mu(\mathcal{F})-1$. Hence (ii).

Suppose $\mathcal{F}$ is compressed and $[n] \notin \mathcal{F}$. Let $M$ be an $\mathcal{E}$-maximal set in $\mathcal{E}$. Suppose $|M|<\mu(\mathcal{F})$. Then there exists a set $M^{\prime} \in \mathcal{F}(n)$ such that $M \subset M^{\prime}$. Since $[n] \notin \mathcal{F}$, $X:=[n] \backslash M^{\prime} \neq \emptyset$. Let $x \in X$ and $M^{\prime \prime}:=\delta_{x, n}\left(M^{\prime}\right)=\left(M^{\prime} \backslash\{n\}\right) \cup\{x\}$. Since $\mathcal{F}$ is compressed, $M^{\prime \prime} \in \mathcal{F}$. But then $M \subsetneq M^{\prime \prime} \in \mathcal{E}$, a contradiction (as $M$ is $\mathcal{E}$-maximal). So $|M| \geqslant \mu(\mathcal{F})$. Hence (iii).

We remark that the inequalities above cannot be replaced by equalities. An example for (iii) is that if $n \geqslant 3$ and $\mathcal{F}$ is the compressed (hereditary) family $2^{[n-1]} \cup 2^{[n-3] \cup\{n\}}$, then $\mu(\mathcal{E})=n-1>n-2=\mu(\mathcal{F})$.

We shall say that a family $\mathcal{F} \subseteq 2^{[n]}$ is quasi-compressed if $\delta_{i, j}(F) \in \mathcal{F}$ for any $F \in$ $\mathcal{F}$ and any $i, j \in U(\mathcal{F})$ with $i<j$. Therefore a quasi-compressed family $\mathcal{F} \subseteq 2^{[n]}$ is isomorphic to a compressed sub-family of $2^{[U(\mathcal{F}) \mid]}$, and the isomorphism is induced by the bijection $\beta: U(\mathcal{F}) \rightarrow[|U(\mathcal{F})|]$ defined by $\beta\left(u_{i}\right):=i, i=1, \ldots,|U(\mathcal{F})|$, where $\left\{u_{1}, \ldots, u_{|U(\mathcal{F})|}\right\}=U(\mathcal{F})$ and $u_{1}<\ldots<u_{|U(\mathcal{F})|}$.

The next lemma is straightforward, so we omit its proof.

Lemma 3.3 Let $\mathcal{H} \subseteq 2^{[n]}$ and $a \in[n]$.

(i) If $\mathcal{H}$ is hereditary, then $\mathcal{H} \backslash \mathcal{H}(a)$ and $\mathcal{H}\langle a\rangle$ are hereditary.

(ii) If $\mathcal{H}$ is quasi-compressed, then $\mathcal{H} \backslash \mathcal{H}(a)$ and $\mathcal{H}\langle a\rangle$ are quasi-compressed.

We shall frequently use the following property of quasi-compressed families.

Lemma 3.4 Let $\mathcal{F} \subseteq 2^{[n]}$ be a quasi-compressed family with $U(\mathcal{F}) \neq \emptyset$. Let $Z \subseteq[n]$ and let $i, j \in U(\mathcal{F}), i \leqslant j$. Then $|\mathcal{F}(Z)| \leqslant\left|\mathcal{F}\left(\delta_{i, j}(Z)\right)\right|$. 
Proof. Let $Y:=\delta_{i, j}(Z)$. Suppose $Y \neq Z$, and let $W:=Z \cap Y$. Then $i<j$, $Z=W \cup\{j\} \neq W$ and $Y=W \cup\{i\} \neq W$. Let $\mathcal{D}:=\{F \in \mathcal{F}: i \in F, F \cap W=\emptyset\}$, $\mathcal{E}:=\{F \in \mathcal{F}: j \in F, F \cap W=\emptyset\}$. Since $\mathcal{F}$ is quasi-compressed and $i, j \in U(\mathcal{F})$, we have $\Delta_{i, j}(\mathcal{E} \backslash \mathcal{E}(i)) \subseteq \mathcal{D} \backslash \mathcal{D}(j)$; so $|\mathcal{D} \backslash \mathcal{D}(j)| \geqslant\left|\Delta_{i, j}(\mathcal{E} \backslash \mathcal{E}(i))\right|=|\mathcal{E} \backslash \mathcal{E}(i)|$. Note that $\mathcal{D}(j)=\mathcal{E}(i)$. Thus, since $|\mathcal{F}(Y)|-|\mathcal{F}(Z)|=(|\mathcal{F}(W)|+|\mathcal{D}|)-(|\mathcal{F}(W)|+|\mathcal{E}|)=$ $(|\mathcal{D}(j)|+|\mathcal{D} \backslash \mathcal{D}(j)|)-(|\mathcal{E}(i)|+|\mathcal{E} \backslash \mathcal{E}(i)|)=|\mathcal{D} \backslash \mathcal{D}(j)|-|\mathcal{E} \backslash \mathcal{E}(i)| \geqslant 0$, the result follows.

For a set $X:=\left\{x_{1}, \ldots, x_{n}\right\} \subset \mathbb{N}$ with $x_{1}<\ldots<x_{n}$ and $r \in[n]$, call $\left\{x_{1}, \ldots, x_{r}\right\}$ the initial $r$-segment of $X$. For convenience, we call $\emptyset$ the initial 0 -segment of $X$.

Corollary 3.5 Let $\mathcal{F} \subseteq 2^{[n]}$ be quasi-compressed. Let $\emptyset \neq Z \subseteq[n]$ and let $Y \in\left(\begin{array}{l}{[n]} \\ |Z|\end{array}\right)$ such that $Y$ contains the initial $|Z \cap U(\mathcal{F})|$-segment of $U(\mathcal{F})$. Then $|\mathcal{F}(Z)| \leqslant|\mathcal{F}(Y)|$.

Proof. Let $Z^{\prime}:=Z \cap U(\mathcal{F})$. If $Z^{\prime}=\emptyset$ then $|\mathcal{F}(Z)|=0 \leqslant|\mathcal{F}(Y)|$. Suppose $Z^{\prime} \neq \emptyset$. Let $Y^{\prime}$ be the initial $\left|Z^{\prime}\right|$-segment of $U(\mathcal{F})$. Since $\mathcal{F}$ is quasi-compressed and $Z^{\prime} \subseteq U(\mathcal{F})$, we can construct a composition of compressions $\delta_{i, j}$ with $i, j \in U(\mathcal{F}), i \leqslant j$, that yields $Y^{\prime}$ when applied on $Z^{\prime}$. Thus $\left|\mathcal{F}\left(Z^{\prime}\right)\right| \leqslant\left|\mathcal{F}\left(Y^{\prime}\right)\right|$ by repeated application of Lemma 3.4. Since $Y^{\prime} \subseteq Y$ and $|\mathcal{F}(Z)|=\left|\mathcal{F}\left(Z^{\prime}\right)\right|$, we have $|\mathcal{F}(Z)| \leqslant\left|\mathcal{F}\left(Y^{\prime}\right)\right| \leqslant|\mathcal{F}(Y)|$.

The following is a well-known fundamental property of compressions that emerged in [4] and that is not difficult to prove.

Lemma 3.6 If $\mathcal{A} \subset 2^{[n]}$ is intersecting and $i, j \in[n]$, then $\Delta_{i, j}(\mathcal{A})$ is intersecting.

\section{Proof of Theorem 1.2}

Lemma 4.1 Let $r, s, n$ and $\mathcal{H}$ be as in Theorem 1.2, and let $(\mathcal{A}, \mathcal{B})$ be a cip with $\emptyset \neq$ $\mathcal{A} \subset \mathcal{H}^{(r)}$ and $\emptyset \neq \mathcal{A} \subset \mathcal{H}^{(s)}$. Let $1 \leqslant i<j \leqslant n$. Then:

(i) $\Delta_{i, j}(\mathcal{A})$ and $\Delta_{i, j}(\mathcal{B})$ are cross-intersecting;

(ii) if either $\Delta_{m, n}(\mathcal{A})=\mathcal{A}$ for all $m \in[n-1]$ or $\Delta_{m, n}(\mathcal{B})=\mathcal{B}$ for all $m \in[n-1]$, then $(A \cap B) \backslash\{n\} \neq \emptyset$ for any $A \in \mathcal{A}$ and $B \in \mathcal{B}$.

Proof. Let $\mathcal{A}^{\prime}:=\{A \cup\{n+1\}: A \in \mathcal{A}\}, \mathcal{A}^{\prime \prime}:=\left\{A^{*} \cup\{n+1\}: A^{*} \in \Delta_{i, j}(\mathcal{A})\right\}$, $\mathcal{B}^{\prime}:=\{B \cup\{n+2\}: B \in \mathcal{B}\}, \mathcal{B}^{\prime \prime}:=\left\{B^{*} \cup\{n+2\}: B^{*} \in \Delta_{i, j}(\mathcal{B})\right\}$. Clearly, the family $\mathcal{C}:=\mathcal{A}^{\prime} \cup \mathcal{B}^{\prime}$ is intersecting, and hence $\Delta_{i, j}(\mathcal{C})$ is intersecting by Lemma 3.6. Since $\Delta_{i, j}(\mathcal{C})=\mathcal{A}^{\prime \prime} \cup \mathcal{B}^{\prime \prime},(\mathrm{i})$ clearly follows.

Suppose without loss of generality that $\Delta_{m, n}(\mathcal{A})=\mathcal{A}$ for all $m \in[n-1]$. Suppose $A \cap B=\{n\}$ for some $A \in \mathcal{A}$ and $B \in \mathcal{B}$. Then, since $|(A \cup B) \backslash\{n\}|=r+s-2<n-1$, the set $X:=[n-1] \backslash(A \cup B)$ is non-empty. Let $x \in X$. Since $\Delta_{x, n}(\mathcal{A})=\mathcal{A}, \delta_{x, n}(A) \in \mathcal{A}$. But $\delta_{x, n}(A) \cap B=\emptyset$, a contradiction. Hence (ii).

Proof of Theorem 1.2. Let $R:=[r]$ and let $\left(\mathcal{A}_{0}, \mathcal{B}_{0}\right)$ be the simple cip $\left(\{R\}, \mathcal{H}^{(s)}(R)\right)$. We clearly have $[\mu(\mathcal{H})] \in \mathcal{H}$ (since $\mathcal{H}$ is compressed) and hence

$$
2^{[\mu(\mathcal{H})]} \subseteq \mathcal{H}
$$


(since $\mathcal{H}$ is hereditary). So $R \in \mathcal{H}^{(r)}$. We therefore have $\emptyset \neq \mathcal{A}_{0} \subset \mathcal{H}^{(r)}$ and $\emptyset \neq \mathcal{B}_{0} \subset \mathcal{H}^{(s)}$. It remains to show that $|\mathcal{A}|+|\mathcal{B}| \leqslant\left|\mathcal{A}_{0}\right|+\left|\mathcal{B}_{0}\right|$ for any $\operatorname{cip}(\mathcal{A}, \mathcal{B})$ with $\emptyset \neq \mathcal{A} \subset \mathcal{H}^{(r)}$ and $\emptyset \neq \mathcal{B} \subset \mathcal{H}^{(s)}$, and we do this using induction on $r$.

Consider the base case $r=1$. By Theorem 1.4, there exists a (single-element) set $Z \in \mathcal{H}^{(1)}$ such that $\left(\{Z\}, \mathcal{H}^{(s)}(Z)\right) \in C(\mathcal{H}, 1, s)$ and hence $|\mathcal{A}|+|\mathcal{B}| \leqslant 1+\left|\mathcal{H}^{(s)}(Z)\right|$. By Corollary 3.5, $\left|\mathcal{H}^{(s)}(Z)\right| \leqslant\left|\mathcal{B}_{0}\right|$. So $|\mathcal{A}|+|\mathcal{B}| \leqslant\left|\mathcal{A}_{0}\right|+\left|\mathcal{B}_{0}\right|$.

Now consider $r \geqslant 2$. Suppose $n=r+s$. So $\mu(\mathcal{H})=n$ and hence $[n] \in \mathcal{H}$. Thus, since $\mathcal{H}$ is hereditary, $\mathcal{H}^{(p)}=\left(\begin{array}{c}{[n]} \\ p\end{array}\right)$ for each $p \in[n]$. Having $n=r+s$ means that for every $A \in\left(\begin{array}{c}{[n]} \\ r\end{array}\right)$ there is only one set $B \in\left(\begin{array}{c}{[n]} \\ s\end{array}\right)$ such that $A \cap B=\emptyset$, so $|\mathcal{A}|+|\mathcal{B}| \leqslant$ $|\mathcal{A}|+\left(\left(\begin{array}{l}n \\ s\end{array}\right)-|\mathcal{A}|\right)=\left|\mathcal{A}_{0}\right|+\left|\mathcal{B}_{0}\right|$.

We now consider $n \geqslant r+s+1$ and proceed by induction on $n$. Let $n^{\prime}:=n-1$.

In view of Lemma 4.1(i) and the assumption that $\mathcal{H}$ is compressed, if $\Delta_{m, n}(\mathcal{A}) \neq \mathcal{A}$ or $\Delta_{m, n}(\mathcal{B}) \neq \mathcal{B}$ for some $m \in[n-1]$, then we can replace $\mathcal{A}$ and $\mathcal{B}$ by $\mathcal{A}^{\prime}:=\Delta_{m, n}(\mathcal{A})$ and $\mathcal{B}^{\prime}:=\Delta_{m, n}(\mathcal{B})$, respectively, and repeat the procedure until we obtain families $\mathcal{A}^{*} \subset \mathcal{H}^{(r)}$ and $\mathcal{B}^{*} \subset \mathcal{H}^{(s)}$ such that $\Delta_{m, n}\left(\mathcal{A}^{*}\right)=\mathcal{A}^{*}$ and $\Delta_{m, n}\left(\mathcal{B}^{*}\right)=\mathcal{B}^{*}$ for all $m \in[n-1]$ (it is well-known and easy to see that such a procedure indeed takes a finite number of steps). We can therefore assume that

$$
\Delta_{m, n}(\mathcal{A})=\mathcal{A} \text { and } \Delta_{m, n}(\mathcal{B})=\mathcal{B} \text { for all } m \in[n-1] .
$$

Thus, by Lemma 4.1(ii),

$$
(A \cap B) \backslash\{n\} \neq \emptyset \text { for any } A \in \mathcal{A} \text { and } B \in \mathcal{B} \text {. }
$$

Let $\mathcal{I}:=\mathcal{H} \backslash \mathcal{H}(n)=\{H \in \mathcal{H}: n \notin H\}$. Similarly, let $\mathcal{C}:=\mathcal{A} \backslash \mathcal{A}(n), \mathcal{D}:=\mathcal{B} \backslash \mathcal{B}(n)$, $\mathcal{E}:=\mathcal{B}_{0} \backslash \mathcal{B}_{0}(n)$. So $\mathcal{C} \subset \mathcal{I}^{(r)}$ and $\mathcal{D}, \mathcal{E} \subset \mathcal{I}^{(s)}$. Note that $\mathcal{C} \neq \emptyset$ and $\mathcal{D} \neq \emptyset$ by (3). Since $\mathcal{H}$ is hereditary, if $[n] \in \mathcal{H}$ then $\mu(\mathcal{I})=n-1$. Thus, if $[n] \in \mathcal{H}$ then $\mu(\mathcal{I}) \geqslant r+s$, and if $[n] \notin \mathcal{H}$ then, since $\mu(\mathcal{H}) \geqslant r+s$, it follows by Lemma 3.2(iii) that $\mu(\mathcal{I}) \geqslant r+s$. Clearly $\mathcal{I}$ is a compressed hereditary sub-family of $2^{[n-1]}$. Therefore, by the inductive hypothesis,

$$
|\mathcal{C}|+|\mathcal{D}| \leqslant\left|\mathcal{A}_{0}\right|+|\mathcal{E}|
$$

Let $\mathcal{J}:=\mathcal{H}\langle n\rangle$. Clearly $\mathcal{J}$ is a compressed hereditary sub-family of $2^{[n-1]}$, and $\mu(\mathcal{J}) \geqslant$ $\mu(\mathcal{H})-1$ by Lemma 3.2(i). Let $r^{\prime}:=r-1$ and $s^{\prime}:=s-1$. So

$$
r^{\prime} \leqslant s^{\prime} \quad \text { and } \quad \mu(\mathcal{J}) \geqslant \mu(\mathcal{H})-1 \geqslant r+s-1>r^{\prime}+s^{\prime} .
$$

We have $\mathcal{A}\langle n\rangle \subset \mathcal{J}^{\left(r^{\prime}\right)}$ and $\mathcal{B}\langle n\rangle \subset \mathcal{J}^{\left(s^{\prime}\right)}$. By (4), $\mathcal{A}\langle n\rangle$ and $\mathcal{B}\langle n\rangle$ are cross-intersecting.

Suppose $\mathcal{A}\langle n\rangle \neq \emptyset$ and $\mathcal{B}\langle n\rangle \neq \emptyset$. Let $R^{\prime}:=\left[r^{\prime}\right]=R \backslash\{r\}$. By the inductive hypothesis, $|\mathcal{A}\langle n\rangle|+|\mathcal{B}\langle n\rangle| \leqslant 1+\left|\mathcal{J}^{\left(s^{\prime}\right)}\left(R^{\prime}\right)\right|$. Similarly to $(2), 2^{[\mu(\mathcal{J})]} \subseteq \mathcal{J}$; so $\left.{ }^{([\mu(\mathcal{J})]} \underset{s^{\prime}}{ }\right) \subseteq \mathcal{J}^{\left(s^{\prime}\right)}$. Since $\mathcal{B}_{0}\langle n\rangle=\mathcal{J}^{\left(s^{\prime}\right)}(R)$,

$$
\begin{aligned}
\left|\mathcal{B}_{0}\langle n\rangle\right| & =\left|\mathcal{J}^{\left(s^{\prime}\right)}\left(R^{\prime}\right)\right|+\left|\left\{B \in \mathcal{J}^{\left(s^{\prime}\right)}: B \cap R^{\prime}=\emptyset, r \in B\right\}\right| \\
& \geqslant\left|\mathcal{J}^{\left(s^{\prime}\right)}\left(R^{\prime}\right)\right|+\left|\left\{B \in\left(\begin{array}{c}
{[\mu(\mathcal{J})] \backslash R^{\prime}} \\
s^{\prime}
\end{array}\right): r \in B\right\}\right|=\left|\mathcal{J}^{\left(s^{\prime}\right)}\left(R^{\prime}\right)\right|+\left(\begin{array}{c}
\mu(\mathcal{J})-r^{\prime}-1 \\
s^{\prime}-1
\end{array}\right)
\end{aligned}
$$


and hence, by $(6),\left|\mathcal{B}_{0}\langle n\rangle\right| \geqslant\left|\mathcal{J}^{\left(s^{\prime}\right)}\left(R^{\prime}\right)\right|+1$. So $|\mathcal{A}\langle n\rangle|+|\mathcal{B}\langle n\rangle| \leqslant\left|\mathcal{B}_{0}\langle n\rangle\right|$. Since $|\mathcal{A}|+|\mathcal{B}|=$ $|\mathcal{C}|+|\mathcal{D}|+|\mathcal{A}\langle n\rangle|+|\mathcal{B}\langle n\rangle|,(5)$ and the last inequality give us $|\mathcal{A}|+|\mathcal{B}| \leqslant\left|\mathcal{A}_{0}\right|+|\mathcal{E}|+\left|\mathcal{B}_{0}\langle n\rangle\right|=$ $\left|\mathcal{A}_{0}\right|+\left|\mathcal{B}_{0}\right|$.

Next, suppose $\mathcal{A}\langle n\rangle=\emptyset$. Let $A \in \mathcal{C}$ (recall that $\mathcal{C} \neq \emptyset$ ). By $(4),|\mathcal{B}\langle n\rangle| \leqslant\left|\mathcal{J}^{\left(s^{\prime}\right)}(A)\right|$. It is easy to see that $U\left(\mathcal{J}^{\left(s^{\prime}\right)}\right)=[l]$ for some $l \in\left[n^{\prime}\right]$ (since $\mathcal{J}$ is compressed); so $\left|\mathcal{J}^{\left(s^{\prime}\right)}(A)\right| \leqslant$ $\left|\mathcal{J}^{\left(s^{\prime}\right)}(R)\right|$ by Corollary 3.5. Since $|\mathcal{A}|+|\mathcal{B}|=|\mathcal{C}|+|\mathcal{D}|+|\mathcal{A}\langle n\rangle|+|\mathcal{B}\langle n\rangle|$, where $\mathcal{A}\langle n\rangle=\emptyset$ and $|\mathcal{B}\langle n\rangle| \leqslant\left|\mathcal{J}^{\left(s^{\prime}\right)}(R)\right|=\left|\mathcal{B}_{0}\langle n\rangle\right|$, it follows by (5) that $|\mathcal{A}|+|\mathcal{B}| \leqslant\left|\mathcal{A}_{0}\right|+\left|\mathcal{B}_{0}\right|$.

Finally, suppose $\mathcal{B}\langle n\rangle=\emptyset$. If $r^{\prime}=s^{\prime}$ (i.e. $r=s$ ) then $|\mathcal{A}|+|\mathcal{B}| \leqslant\left|\mathcal{A}_{0}\right|+\left|\mathcal{B}_{0}\right|$ follows by an argument similar to the one for the previous case. Suppose $r^{\prime}<s^{\prime}$. Let $\mathcal{K}_{0}:=\mathcal{J} \backslash \mathcal{J}(1):=\{J \in \mathcal{J}: 1 \notin J\}$ and $\mathcal{K}_{1}:=\mathcal{J}\langle 1\rangle$. So $\mathcal{K}_{0}, \mathcal{K}_{1} \subseteq 2^{[2, n-1]}$. By Lemma 3.3, $\mathcal{K}_{0}$ and $\mathcal{K}_{1}$ are hereditary and quasi-compressed. By (i) and (ii) of Lemma 3.2, $\mu\left(\mathcal{K}_{0}\right) \geqslant$ $\mu(\mathcal{J})-1$ and $\mu\left(\mathcal{K}_{1}\right) \geqslant \mu(\mathcal{J})-1$. Thus, by $(6), \mu\left(\mathcal{K}_{0}\right) \geqslant r^{\prime}+s^{\prime}$. Let $R^{*}:=[2, r]$ and $S^{*}:=[2, s]$. It is clear from (2) that $R^{*}, S^{*} \in \mathcal{K}_{0}$. Note that therefore $R^{*}$ and $S^{*}$ are initial segments of $U\left(\mathcal{K}_{0}\right)$. Since $\left(\mathcal{K}_{0}^{\left(r^{\prime}\right)}\left(S^{*}\right),\left\{S^{*}\right\}\right)$ is a cip with the first family contained in $\mathcal{K}_{0}^{\left(r^{\prime}\right)}$ and the second family contained in $\mathcal{K}_{0}^{\left(s^{\prime}\right)}$, the inductive hypothesis gives us $\left|\mathcal{K}_{0}^{\left(r^{\prime}\right)}\left(S^{*}\right)\right|+\left|\left\{S^{*}\right\}\right| \leqslant\left|\left\{R^{*}\right\}\right|+\left|\mathcal{K}_{0}^{\left(s^{\prime}\right)}\left(R^{*}\right)\right|$ and hence

$$
\left|\mathcal{K}_{0}^{\left(r^{\prime}\right)}\left(S^{*}\right)\right| \leqslant\left|\mathcal{K}_{0}^{\left(s^{\prime}\right)}\left(R^{*}\right)\right| .
$$

Let $\mathcal{L}_{0}:=\left\{A \in \mathcal{J}^{\left(r^{\prime}\right)}(S): 1 \notin A\right\}$ and $\mathcal{L}_{1}:=\left\{A \backslash\{1\}: 1 \in A \in \mathcal{J}^{\left(r^{\prime}\right)}(S)\right\}$. Let $\mathcal{M}_{0}:=$ $\left\{B \in \mathcal{B}_{0}\langle n\rangle: 1 \notin B\right\}$ and $\mathcal{M}_{1}:=\left\{B \backslash\{1\}: 1 \in B \in \mathcal{B}_{0}\langle n\rangle\right\}$. Note that $\mathcal{L}_{0}=\mathcal{K}_{0}^{\left(r^{\prime}\right)}\left(S^{*}\right)$ and $\mathcal{M}_{0}=\mathcal{K}_{0}^{\left(s^{\prime}\right)}\left(R^{*}\right)$. So $\left|\mathcal{L}_{0}\right| \leqslant\left|\mathcal{M}_{0}\right|$ by $(7)$. Let $r^{\prime \prime}:=r^{\prime}-1$ and $s^{\prime \prime}:=s^{\prime}-1$. Similarly to $(6), \mu\left(\mathcal{K}_{1}\right)>r^{\prime \prime}+s^{\prime \prime}$. By Lemma 3.1, $\left|\mathcal{K}_{1}^{\left(r^{\prime \prime}\right)}\right|<\left|\mathcal{K}_{1}^{\left(s^{\prime \prime}\right)}\right|$. Thus, since $\mathcal{L}_{1}=\mathcal{K}_{1}^{\left(r^{\prime \prime}\right)}$ and $\mathcal{M}_{1}=\mathcal{K}_{1}^{\left(s^{\prime \prime}\right)},\left|\mathcal{L}_{1}\right|<\left|\mathcal{M}_{1}\right|$. We therefore have

$$
\left|\mathcal{J}^{\left(r^{\prime}\right)}(S)\right|=\left|\mathcal{L}_{0}\right|+\left|\mathcal{L}_{1}\right|<\left|\mathcal{M}_{0}\right|+\left|\mathcal{M}_{1}\right|=\left|\mathcal{B}_{0}\langle n\rangle\right| .
$$

Now let $D \in \mathcal{D}$. By $(4),|\mathcal{A}\langle n\rangle| \leqslant\left|\mathcal{J}^{\left(r^{\prime}\right)}(D)\right|$. It is easy to see that $U\left(\mathcal{J}^{\left(r^{\prime}\right)}\right)=[l]$ for some $l \in\left[n^{\prime}\right]$ (since $\mathcal{J}$ is compressed); so $\left|\mathcal{J}^{\left(r^{\prime}\right)}(D)\right| \leqslant\left|\mathcal{J}^{\left(r^{\prime}\right)}(S)\right|$ by Corollary 3.5. Thus, by (8), $|\mathcal{A}\langle n\rangle|<\left|\mathcal{B}_{0}\langle n\rangle\right|$. Together with (5) and $\mathcal{B}\langle n\rangle=\emptyset$, this gives us $|\mathcal{A}|+|\mathcal{B}|<\left|\mathcal{A}_{0}\right|+\left|\mathcal{B}_{0}\right|$.

Acknowledgements: The author is indebted to an anonymous referee for checking the paper carefully and suggesting helpful comments.

\section{References}

[1] P. Borg, Extremal t-intersecting sub-families of hereditary families, J. London Math. Soc. 79 (2009) 167-185.

[2] V. Chvátal, Unsolved Problem No. 7, in: C. Berge, D.K. Ray-Chaudhuri (Eds.), Hypergraph Seminar, Lecture Notes in Mathematics, Vol. 411, Springer, Berlin, 1974. 
[3] V. Chvátal, Intersecting families of edges in hypergraphs having the hereditary property, in: C. Berge, D.K. Ray-Chaudhuri (Eds.), Hypergraph Seminar, Lecture Notes in Mathematics, Vol. 411, Springer, Berlin, 1974, pp. 61-66.

[4] P. Erdős, C. Ko and R. Rado, Intersection theorems for systems of finite sets, Quart. J. Math. Oxford (2) 12 (1961) 313-320.

[5] P. Frankl, The shifting technique in extremal set theory, in: C. Whitehead (Ed.), Combinatorial Surveys, Cambridge Univ. Press, London/New York, 1987, pp. 81110.

[6] P. Frankl and N. Tokushige, Some best possible inequalities concerning crossintersecting families, J. Combin. Theory Ser. A 61 (1992) 87-97.

[7] A.J.W. Hilton and E.C. Milner, Some intersection theorems for systems of finite sets, Quart. J. Math. Oxford (2) 18 (1967) 369-384.

[8] G.O.H. Katona, A theorem of finite sets, in: Theory of Graphs, Proc. Colloq. Tihany, Akadémiai Kiadó (1968) 187-207.

[9] J.B. Kruskal, The number of simplices in a complex, in: Mathematical Optimization Techniques, University of California Press, Berkeley, California, 1963, pp. 251-278.

[10] J.E. Simpson, A bipartite Erdôs-Ko-Rado theorem, Discrete Math. 113 (1993) 277280.

[11] H. Snevily, A new result on Chvátal's conjecture, J. Combin. Theory Ser. A 61 (1992) 137-141. 